\title{
Clinical and Economic Benefits of Pharmacist Involvement in a Community Hospital-Affiliated Patient-Centered Medical Home
}

\author{
Meredith L. Tate, PharmD; Sydney Hopper, PharmD; and Sean Paul Bergeron, PharmD
}

\begin{abstract}
BACKGROUND: The primary goals of an accountable care organization (ACO) are to reduce health care spending and increase quality of care. Within an ACO, pharmacists have a unique opportunity to help carry out these goals within patient-centered medical homes (PCMHs). Pharmacy presence is increasing in these integrated care models, but the pharmacist's role and benefit is still being defined.
\end{abstract}

OBJECTIVE: To exhibit the clinical and economic benefit of pharmacist involvement in ACOs and PCMHs as documented by clinical interventions (Cls) and drug cost reductions.

METHODS: This is a retrospective quality improvement study. All interventions made by the pharmacist during the study period were documented using TAV Health. The interventions were then analyzed. Specific identified endpoints included the total number of documented interventions and number of Cls from each category, transition of care (TOC) medication reconciliations performed, discrepancies identified during TOC medication reconciliation, and cost savings generated from generic and therapeutic alternative use. $\mathrm{Cl}$ categories were collaborative drug therapy management, medication therapy management (MTM), medication reconciliation, patient and provider education, and drug cost management.

RESULTS: During the study period (October 2016-March 2017), a pharmacist was in clinic 8 hours per week. Sixty-three patients were included in the study. There were 283 Cls documented, with a majority of the interventions associated with MTM or cost management (94 and $88 \mathrm{Cls}$, respectively). There were 37 education Cls, 36 TOC medication reconciliations performed, and 28 collaborative drug therapy management Cls. From the 36 TOC medication reconciliations, $\mathbf{2 4 0}$ medication discrepancies were found, with a majority associated with medication omission. A cost savings of $\$ 118,409$ was gained from generic and therapeutic alternative substitutions.

CONCLUSIONS: Clinical benefit of pharmacy services was demonstrated through documented Cls. Pharmacists can have a dramatic and quantitative effect on reducing drug costs by recommending less expensive generic or therapeutic alternatives. Documenting Cls allows pharmacists to provide valuable evidence of avoided drug misadventures and identification of medication discrepancies. Such evidence supports an elevated quality of care.

J Manag Care Spec Pharm. 2018;24(2):160-64

Copyright $\odot 2018$, Academy of Managed Care Pharmacy. All rights reserved.

\section{What is already known about this subject}

Many different types of integrated care models exist in which pharmacists manage drug therapy.

A multitude of studies have used documentation of clinical interventions to demonstrate improvements in outcomes, readmission rates, cost savings, and/or quality measures, as well as favorable return on investments with pharmacist-driven programs within integrated health care teams.

Pharmacy presence is currently lacking in the accountable care organization (ACO) model as reported in a 2015 study, which estimated that only $26 \%$ of ACOs include a pharmacy and only $19 \%$ have contracted pharmacy services.

\section{What this study adds}

This study provides further confirmation of the beneficial role of pharmacy within patient-centered medical homes and ACOs.

As more pharmacists become incorporated into integrated care models, this clinic's project may serve as an example for other systems and further define the function of pharmacists within the multidisciplinary team.

This study establishes groundwork for conducting additional research to corroborate the pharmacist's role in increasing quality of care.
A n accountable care organization (ACO) is a group of health care providers that share financial benefits and risks of participating in the Medicare Shared Savings Program (MSSP) or similar contracts with other insurance providers. ACOs are compared against benchmarks intended to assess whether they generate savings or losses for the Medicare program, and they are either rewarded a portion of the savings or held liable for a portion of the losses. In order to generate savings, the ACO must reduce total health care expenditures per patient and increase quality of care. The provider network within an ACO consists of any practitioner or health system that provides care to Medicare beneficiaries. However, a 2015 study reported that only $26 \%$ of ACOs include a pharmacy, and only 19\% have contracted pharmacy services. ${ }^{1}$ This study revealed new opportunities for pharmacist involvement by combining the goals and the inherent multidisciplinary model of the ACO. Pharmacists can play a vital role in reducing drug spending and providing quality medication therapy management (MTM). ${ }^{1,2}$ 
A patient-centered medical home (PCMH) is a model of care that a practice may voluntarily adopt, which offers coordinated, comprehensive, and integrated care and offers patients more involvement in their own care. To become a $\mathrm{PCMH}$, a practice must be certified by an organization such as the National Committee for Quality and Assurance. Data have shown that certified PCMHs can provide improved outcomes, quality of care, and patient experience, as well as reduce costs. Payers also offer enhanced reimbursement and incentives for having a PCMH certification. ${ }^{3}$ The role of a pharmacist in a PCMH is the same as that in an ACO: helping the organization to meet benchmarks set within the MSSP. To do so, pharmacists often provide collaborative care, MTM, and patient and provider education and manage drug costs and medication reconciliation.

Many different types of integrated care models exist in which pharmacists manage drug therapy. A literature search revealed a multitude of studies demonstrating improvements in outcomes, readmission rates, cost savings, and/or quality measures, as well as favorable return on investments with pharmacist-driven programs within integrated health care teams. . $^{4-13}$ The lack of pharmacy presence in many ACOs may be a result of several challenges, including difficulty coordinating and communicating across the health care continuum, identifying a specific population group that would benefit from pharmacy services, and lack of provider status in many states. ${ }^{1}$ However, demonstrating value by measuring the quality and financial effect of pharmacy-driven services may be the largest barrier because of the inability to bill for services and the expensive salary of today's pharmacist. Despite the barriers that other studies have noted, it would be strategic to include pharmacists within the integrated care model in order to optimize medication use as a key component of improving quality and avoiding excessive drug expenditures. ${ }^{14}$

The ambulatory care pilot at $\mathrm{CHI}$ Memorial Hospital was established in 2016 as an initiative between the ACO and pharmacy department in hopes of increasing pharmacy presence in the provider network. Pharmacy services were first piloted in a hospital-affiliated PCMH. The PCMH is an internal medicine clinic with 2 physicians and a nurse practitioner. Services provided by pharmacists were aligned with the goals of the ACO and were focused on collaborative drug therapy management, cost management, transition of care (TOC) medication reconciliation, MTM, and patient and provider education. The pharmacist was employed through the ACO, so the scope of practice was limited to Medicare beneficiaries and $\mathrm{CHI}$ Memorial employees with contracted Blue Cross Blue Shield employee insurance.

The purpose of this study was to exhibit the clinical and economic value of pharmacist involvement within the $\mathrm{PCMH}$ by quantifying cost savings and documenting clinical interventions (CIs). The data collected from this study will be used to support and expand the role of the pharmacist within the ACO.

\section{Methods}

\section{Study Design}

This is a single-center, retrospective, quality improvement study conducted in a 3-provider internal medicine clinic with PCMH certification. Within the pre-existing structure of the clinic, an ambulatory care pharmacist provided several services for the PCMH under partnership with the ACO that included but was not limited to collaborative drug therapy management, cost management, TOC medication reconciliation, MTM, and patient and provider education. For each service, the pharmacist recorded any CI using TAV Health, the documentation and communication software used by the PCMH. Patient demographics were also obtained from TAV Health.

MTM patient candidates were obtained through 2 pathways. First, providers consulted the pharmacist to manage a patient's medication and provide recommendations. Second, each patient discharged from the hospital was scheduled for a TOC visit with the physician or nurse practitioner within 7-14 days after discharge, after which the pharmacist made contact with the patient for follow-up and MTM. Immediately following discharge from the hospital, a chart review of the patient's medications was conducted, and a TOC medication reconciliation CI was documented to record the event, along with any recommendations made to the provider. Collaborative drug therapy management CIs were typically recorded after identification of patient needs, and subsequent referrals to a different health care provider (such as a health educator) were on an as needed basis. Any cost management CI was defined as a direct cost savings from switching one medication to another. Assisting patients with medication assistance programs or insurance coverage was also considered a cost management $\mathrm{CI}$, but a numeric cost savings was unable to be calculated for these interventions. If a recommendation led to a switch from one medication to a less expensive medication, the direct cost savings was determined by subtracting the annual cost of the less expensive medication from the more expensive medication based on average wholesale price. Patient or provider education CIs were documented any time the pharmacist presented drug information to a provider or counseled a patient on appropriate medication use.

\section{Study Population}

This study included any adult patient for whom a pharmacist documented a clinical CI and who also had an eligible insurance plan (Medicare or employee Blue Cross Blue Shield). For the TOC services, patients were excluded if they were discharged to a skilled nursing, hospice, rehab, or other facility; were not evaluated by a pharmacist after discharge; or expired before the TOC visit. 


\begin{tabular}{l|c}
\hline \multicolumn{1}{c|}{ TABLE 1 } & Patient Demographics \\
\hline Characteristics & Population (N=63) \\
\hline Female, $\mathrm{n}(\%)$ & $50(79)$ \\
\hline Male, $\mathrm{n}(\%)$ & $13(21)$ \\
\hline Average age, years & 68 \\
\hline Included from TOC & 36 \\
\hline Included from documented CI & 55 \\
\hline CI= clinical intervention; $T O C=$ transition of care. \\
\hline
\end{tabular}

\section{Data Collection Procedures}

For this study, only the number and types of interventions were obtained from TAV Health. For any conversion to a less expensive drug therapy, the names of the drugs were recorded so that cost savings could be extrapolated. Study endpoints included total documented CIs and the number of documented CIs associated with collaborative drug therapy management, MTM, medication reconciliation, patient and provider education, and cost management. Cost savings resulting from a switch to a less expensive medication was totaled as a part of cost management services. For TOC, the total number of medication reconciliations and associated discrepancies were recorded. Data collection began following institutional review board approval in October 2016 and continued through March 2017.

\section{Statistical Analyses}

No statistical analysis was performed, since data were only used to quantify the number of interventions and associated cost savings. Means of central tendency were used to aid in describing patient characteristics.

\section{Data Security}

This study is an observational retrospective analysis with minimal risk. Anticipated problems could include breach of security. HIPAA guidelines were strictly followed to protect patient confidentiality and maintain records. No personally identifiable information was collected during this study. The information collected only included quantity of interventions, types of interventions, and associated cost savings, and the analysis of this data was only used to support and expand pharmacist involvement within the ACO.

\section{Results}

Sixty-three patients were included in this study. Table 1 lists patient demographics. Some patients, who were included for the TOC arm of the study, also had additional CIs documented, so there was some overlap between inclusion populations. Of the patient group, $79 \%$ were female; $21 \%$ were male; and the average age was 68 years (Table 1). There were a total of 283 CIs documented during the study period, which encompassed 192 hours of pharmacist clinic time (equivalent to 24 full-time

\section{FIGURE 1 Number of Documented Clinical} Interventions

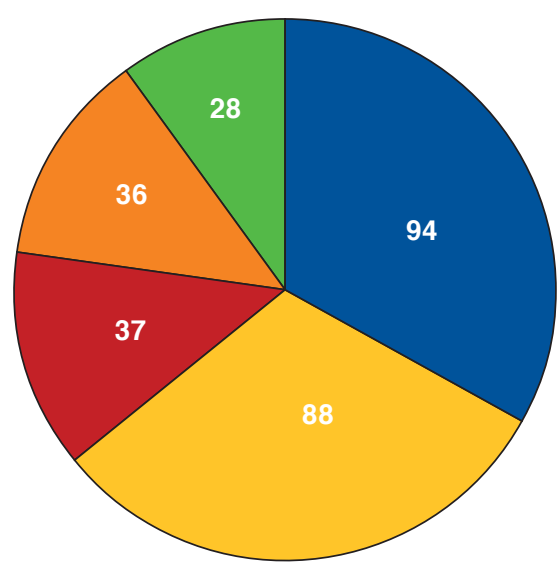

Medication therapy management

Cost management

- Patient and provider education

Medication reconciliation

Collaborative drug therapy management

days). The breakdown of CIs was as follows: 94 (33.2\%) MTM interventions, 88 (31.1\%) cost management interventions, 37 (13.1\%) patient or provider education interventions, 36 (12.7\%) TOC medication reconciliations, and 28 (9.9\%) collaborative drug therapy management interventions (Figure 1). For those interventions associated with cost management, 16 recommendations were made to switch a medication to a less expensive generic or therapeutic alternative, resulting in an extrapolated cost savings of $\$ 118,409$ per year.

For the TOC portion of the study, 36 medication reconciliations were performed following discharge from the hospital and before the TOC appointment at the PCMH. From these medication reconciliations, 240 medication discrepancies were uncovered, including medication duplications and dosing errors, but the vast majority of these discrepancies were medication omissions, that is, a medication was missing from either the inpatient list or the outpatient list. Home medications that were added, changed, or discontinued upon discharge were not considered a medication discrepancy.

\section{Discussion}

MTM constituted the largest portion of interventions. MTM activities primarily included either face-to-face meetings or patient interviews via telephone. During these discussions, medicationrelated issues were often uncovered and resolved. For example, it was discovered that a patient had been taking acyclovir 
chronically and did not know why she was prescribed the medication. Upon discussion with the provider, it was determined that the medication was unnecessary and was subsequently discontinued.

Cost management interventions provided the most convincing evidence of economic benefit. Just shy of $\$ 120,000$ yearly savings were appreciated from recommending a medication switch to either a generic or a less expensive therapeutic alternative. Brand name drugs that were switched to generic included Glumetza, Nuvigil, Vimovo, or Treximet. Examples of therapeutic interchanges included switching fluoxetine tablets to fluoxetine capsules, Silenor to zolpidem, armodafinil to modafinil, or rosuvastatin to atorvastatin. Such switches are noted to be a benefit to the ACO, since it reduces total health care expenditure per patient, which is one of the major benchmarks that must be met in order to receive shared savings. In addition, the pharmacist assisted many patients in acquiring financial aid via medication assistance programs or obtaining proper Medicare Part D insurance plans.

As the pharmacotherapy expert, the pharmacist served as a drug information resource to providers while in clinic. A newsletter was drafted to provide further education, such as commonly prescribed brand name drugs with less expensive generics available and other pharmacy-related health care updates. The newsletter will continue to be published periodically for practitioners and other clinic staff. The pharmacist was a well trusted and respected member of the integrated team and was frequently sought out for recommendations and drug information. Patient education was also a vital role of the pharmacist during MTM or medication reconciliation.

The concerning number of medication discrepancies identified during TOC led to the conclusion that the TOC medication reconciliation process was disjointed and needed improvement. The lack of an electronic health record and communication across the continuum represent barriers that will be difficult to overcome. Increased collaboration between settings is vital, and future directions will be focused on to optimize current resources and overcome existing barriers.

Collaborative patient care plays a central role in the PCMH and ACO models, and we believe that the pharmacist was vital for collaborative drug therapy management. During patient encounters, the pharmacist was able to identify barriers or needs of patients and refer the patients to different resources such as health coaches or case managers. In return, the health coaches in the clinic were able to refer patients to pharmacy services.

\section{Limitations}

There were several limitations to this study. First, the study was conducted during the infancy of the ambulatory care pilot. The pilot underwent many changes and transformations early on, and the pharmacy department, PCMH staff, and ACO are continuously reassessing the needs of all parties and adjusting accordingly. As the program expands, a larger system with a more robust provider population would eliminate the possibility of confounders attributed to personality or other characteristics. Second, a pharmacist was only in clinic 8 hours per week, which limits patient and provider contact time. In addition to limited hours, pharmacists were restricted in patient outreach to Medicare beneficiaries and patients with contracted hospital insurance. Finally, the hospital system lacked a universal electronic health record, and as a result, the systems used by the hospital and the PCMH could not communicate with each other. The lack of a universal system makes a smooth TOC medication reconciliation process extremely difficult and disjointed.

\section{Conclusions}

The presence of pharmacists in an integrated health care team setting such as a PCMH has positive benefits for patients, the clinic, and the ACO. Recommending generic and less expensive therapeutic alternatives provide for a quantitative and dramatic effect on cost savings as a direct result of pharmacist involvement. While the majority of the cost savings was a direct savings to insurance providers, this savings also assisted the ACO in meeting benchmarks by reducing total health care expenditures per patient. In addition, there is a clear clinical benefit of using pharmacists in this setting, as demonstrated by documented CIs, which will ultimately lead to higher quality patient care. CI documentation allows for valuable evidence in the avoidance of adverse drug events or medication errors and identification of medication discrepancies across the TOC process. Finally, closing the gap in the continuum of care is an ongoing process. With a lack of an electronic health record, current resources will need to be optimized in order to further advance the TOC process.

\section{Authors}

MEREDITH L. TATE, PharmD; SYDNEY HOPPER, PharmD; and SEAN PAUL BERGERON, PharmD, CHI Memorial Hospital, Chattanooga, Tennessee.

AUTHOR CORRESPONDENCE: Meredith L. Tate, PharmD, CHI Memorial Hospital, 2525 Desales Ave., Chattanooga, TN 37404.E-mail:mlt0008@auburn.edu.

\section{DISCLOSURES}

No outside funding supported this study. The authors have nothing to disclose.

Study concept and design were contributed by Tate and Hopper, along with Bergeron. Tate collected and interpreted the data, as well wrote the manuscript, which was revised by all the authors. 


\section{REFERENCES}

1. Colla CH, Lewis VA, Beaulleu-Jones BR, Morden NE. Role of pharmacy services in accountable care organizations. J Manag Care Spec Pharm. 2015;21(4):338-44. Available at: http://www.amcp.org/WorkArea/ DownloadAsset.aspx?id=19246.

2. ASHP Task Force on Accountable Care Organizations. Report of the 2012 ASHP Task Force on Accountable Care Organizations. Am J Health Sys Pharm. 2013;70(1):66-76

3. National Committee for Quality and Assurance. Patient-centered medical home (PCMH) recognition. 2017. Available at: http://www.ncqa.org/programs/recognition/practices/patient-centered-medical-home-pcmh. Accessed December 18, 2017.

4. Smith M, Bates DW, Bodenheimer TS. Pharmacists belong in accountable care organizations and intregrated care teams. Health Affairs (Millwood). 2013;32(11):1963-70

5. Amara S, Adamson RT, Lew I, Slonim A. Accountable care organizations: impact on pharmacy. Hosp Pharm. 2014:49(3):253-59.

6. Elman S, Zaiken K. Improving performance of an accountable care organization on a quality measure assessing $\beta$-blocker use in systolic heart failure. Am J Health Syst Pharm. 2016;73(17 Suppl 4):S121-25.

7. Alhossan A, Kennedy A, Leal S. Outcomes of annual wellness visits provided by pharmacists in an accountable care organization associated with a federally qualified health center. Am J Health Syst Pharm. 2016;73(4):225-28.
8. Brummel A, Lustiq A, Westrich K, et al. Best practices: improving patient outcomes and costs in an ACO through comprehensive medication therapy management. J Manag Care Spec Pharm. 2014;20(12):1152-58. Available at: http://www.jmcp.org/doi/abs/10.18553/jmcp.2014.20.12.1152.

9. Schnur ES, Adams AJ, Klepser DG, et al. PCMHs, ACOs, and medication management: lessons learned from early research partnerships. J Manag Care Pharm. 2014;20(2)201-05. Available at: http://www.jmcp.org/doi/10.18553/ jmcp.2014.20.2.201.

10. Smith M, Giuliano MR, Starkowski MP. In Connecticut: improving patient medication management in primary care. Health Affairs (Millwood). 2011;30(4):646-54.

11. Isetts BJ, Brummel AR, de Oliveira DR, Moen DW. Managing drug-related morbidity and mortality in the patient-centered medical home. Med Care. 2012;50(11):997-1001.

12. Ramalho de Oliveira D, Brummel AR, Miller DB. Medication therapy management: 10 years of experience in a large integrated health care system. J Manag Care Pharm. 2010;16(3):185-95. Available at: http://www.jmcp.org/ doi/10.18553/jmcp.2010.16.3.185.

13. Choe HM, Farris KB, Stevenson JG, et al. Patient-centered medical home: developing, expanding, and sustaining a role for pharmacists. Am J Health Syst Pharm. 2012;69(12):1063-71.

14. Joseph T, Hale GM, Eltaki SM, et al. Integration strategies of pharmacists in primary care-based accountable care organizations: a report from the Accountable Care Organization Research Network, Services, and Education. J Manag Care Spec Pharm. 2017;23(5):541-48. Available at: http://www.jmcp. org/doi/10.18553/jmcp.2017.23.5.541. 